\title{
Improving patient safety by enhancing raising concerns at medical school: a curriculum review
}

\author{
Authors: Faye Gishen, Luke Johnson, Natasha Malik, Irene Gafson, Naomi Gostelow, Jayne Kavanagh and \\ Ann Griffin
}

\begin{abstract}
Aims
Raising concerns (RC) as a tool for improving patient safety has been researched among qualified doctors, but while medical students frequently encounter unprofessional behaviours, little is known about the barriers to, and the culture of, RC among medical undergraduates, which this study was first to address.
\end{abstract}

\section{Methods}

This work was led by a final-year medical student in conjunction with the faculty. A university student-staff collaboration faculty development grant funded the work. Ethics was obtained through the local research ethics committee (REC).

We wanted to ascertain the opinions and experiences of medical students relating to $\mathrm{RC}$. The authors conducted a mixed methods quantitative and qualitative research study over a 9-month period. Research was based at a UK medical school and involved data collection using an anonymous, voluntary survey distributed to all medical students (replies, $n=363$ ) and voluntary attendance focus group work ( $n=24$ ) recruited by email. Both tools investigated student attitudes towards RC and explored student ideas for solutions to improving the process and their engagement. The focus group data was thematically analysed by three researchers.

\section{Results}

The authors identified five key themes which described medical student opinions on RC (process of RC, nature of RC, barriers to RC, suggestions for improvement and parallels to the NHS).

Challenges to RC fell under the following.

> Comprehension; understanding when it is appropriate to raise a concern and how to do so.

> Conviction; understanding why it is important and recognising this as a moral responsibility.

> Courage; having the resilience to overcome fear and manage oneself in a situation where a concern is raised.

The data were used to improve the RC curriculum and access to the RC system, with the aims of improving patient safety, the patient experience and the student experience. We adapted our RC curriculum by embedding small group work (year 1), a lecture (year 4) and a Schwartz Round (year 5; a large confidential reflective practice forum to discuss the emotional aspects of healthcare).

\section{Conclusion}

Failure of RC by healthcare workers may compromise patient safety. This research explores these issues in medical students and discusses some innovations in the medical undergraduate curriculum, offering a good practice model for other medical and healthcare curricula. Despite being a single study in one UK medical school, we propose changes which may inspire other educators to build upon their RC curricula to foster more transparent undergraduate cultures, and ultimately improve patient experience and safety.

\section{Conflict of interest statement}

No conflict of interest declared. 